\title{
Tailoring the scattering properties of coding metamaterials based on machine learning
}

\author{
Shuai Yang, Kuang Zhang, Xumin Ding, Guohui Yang, and Qun Wu* \\ School of Electronic \& Information Engineering, Harbin Institute of Technology, Harbin, PR China
}

Received: 18 April 2021 / Accepted: 18 April 2021

\begin{abstract}
Diverse electromagnetic (EM) responses of coding metamaterials have been investigated, and the general research method is to use full-wave simulation. But if we only care its scattering properties, it is not necessary to perform full-wave simulation, which is usually time-consuming. Machine learning has significantly impelled the development of automatic design and optimize coding matrix. Based on metamaterial particle that has multiple response and genetic algorithm which is coupled with the scattering pattern analysis, we can optimize the coding matrix quickly to tailor the scattering properties without conducting full-wave simulation a lot of times for optimization. Since the coding matrix control of each particle allow modulation of EM wave, various EM phenomena can be achieved easier. In this paper, we proposed two reflective unitcells with different reflection phase, and then a semi-analytical model is built up for unitcells. To tailor the scattering properties, genetic algorithm normally based on binary coding, is coupled with the scattering pattern analysis in order to optimize the coding matrix. Finally, simulation results are compared with the semi-analytical calculation results and it is found that the simulation results agree very well with the theoretical values.
\end{abstract}

Keywords: Machine learning / coding metamaterials / optimization of coding matrix

\section{Introduction}

Metamaterials, normally composed by artificially periodic structures with sub-wavelength scales, made it possible to design multifunction coding metamaterials [1-3]. Various of extraordinary EM phenomena have been found in microwave, terahertz and optical fields [4-7]. Consequently, coding metamaterials have tremendous application potentials, numerous devices like invisibility cloak, planar lens, and perfect absorber were made by using coding metamaterials [8-10]. Most of aforementioned metamaterials have a specific function, therefore the manipulation of EM wave is fixed once the design is completed. In recent years, much attention has been paid on active coding metamaterials whose EM response can be dynamically controlled [11,12]. While a variety of functionalities have been successfully presented with different metamaterials, the way to get coding matrix and simultaneous demonstration of different functionalities on a certain metamaterial has not been fully reported.

The presented study focuses on the optimization of the coding matrix based on machine learning. Initially, we presented two designs of metamaterial unit cells with $\pi$ phase difference and we simulated the reflection

\footnotetext{
* e-mail: qwu@hit.edu.cn
}

performances using full-wave simulation software. Subsequently we arranged the metamaterial particles periodically to construct a metasurface. Finally, we get certain scattering properties by programming a coding matrix with the model which is constructed under the open-source machine learning framework of TensorFlow [13].

\section{Construction of semianalytical model and tensor model}

First we propose a purpose architecture to automatically model and optimize coding matrix. Figure 1 illustrates the flowchart of the Machine learning based optimization. In what follows, we study the scattering properties of our coding metamaterials for plane-wave illumination. As a meaningful observable to quantify the scattering properties, we consider the bistatic RCS:

$$
\sigma(\theta, \phi)=\lim _{x \rightarrow \infty} 4 \pi r^{2} \frac{\left|E_{s}\right|^{2}}{\left|E_{i}\right|^{2}} .
$$

Accordingly, we define the RCS ratio

$$
\gamma=\frac{\max _{\theta, \phi} \sigma_{M M}(\theta, \phi)}{\max _{\theta, \phi} \sigma_{P E C}(\theta, \phi)}
$$




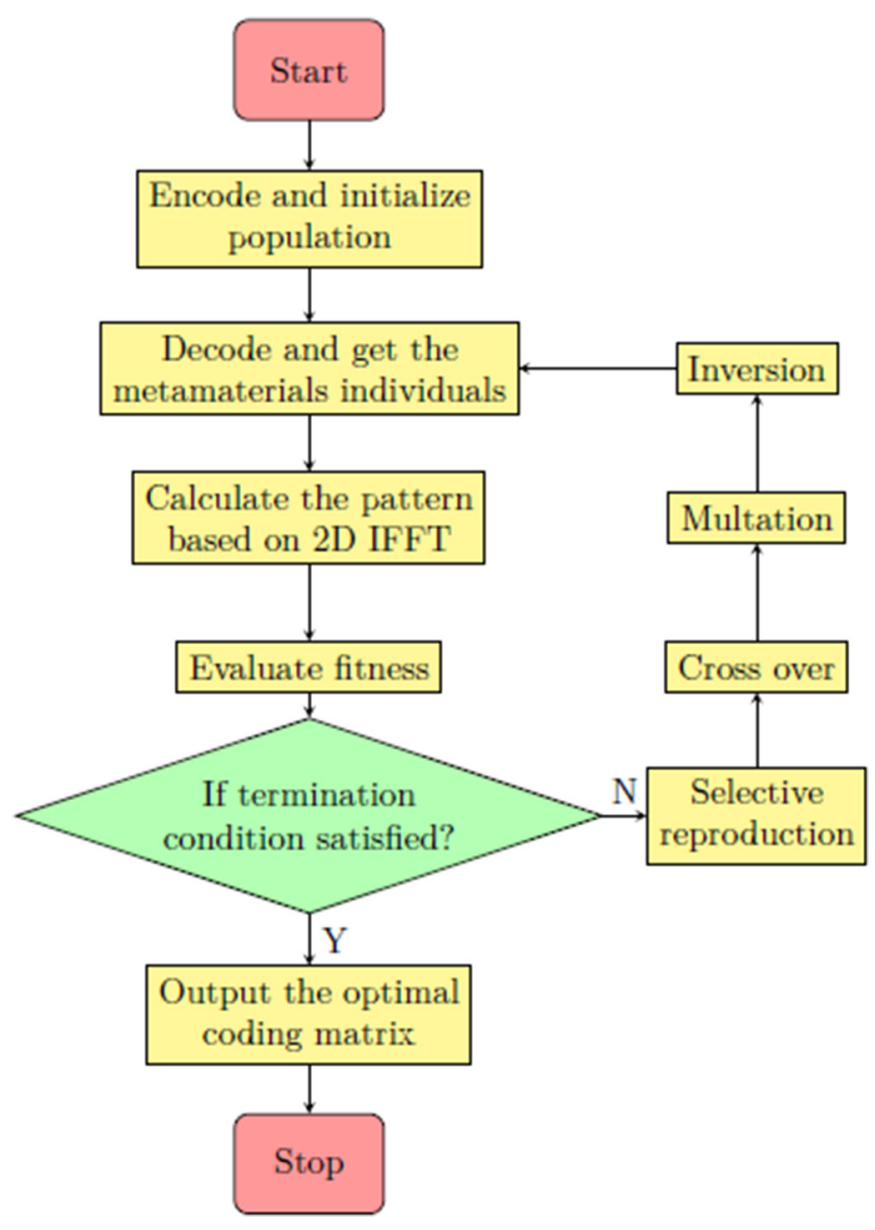

Fig. 1. Flowchart of optimization based generic algorithm.

where $E_{i}$ and $E_{s}$ denote the incident and scattered electric fields respectively, $(\theta, \phi)$ denote the observation direction in the far fields, and the subscripts "MM" and "PEC" indetify the metamaterials and PEC targets. In equation (2) requires the full-wave numerical solution of two 3D scattering problems. Nevertheless, the Semianalytical approximation that was developed in "Coding metamaterials, digital metamaterials and programmable metamaterials" [1]. And the directivity of metamaterials can be described as

$$
D(\theta, \phi)=\frac{4 \pi|f(\theta, \phi)|^{2}|F|^{2}}{\int_{0}^{2 \pi} d \phi \int_{0}^{\frac{\pi}{2}} d \theta|f(\theta, \phi)|^{2}|F|^{2}}
$$

where $F=F(k \sin \theta \cos \phi, k \sin \theta \sin \phi)$. The expression in equation (3) closely resembles the directivity of antenna arrays. So, we considered every unitcells as an antenna.

The proposed machine-learning model for designing coding metamaterials is schematically depicted in Figure 2. As indicated by the orange zone, the model contains tensor module and semianalytical module, where both modules allowing data to flow towards to coding matrix and design pattern. The dataflow in the machine-learning is denoted by the arrows.

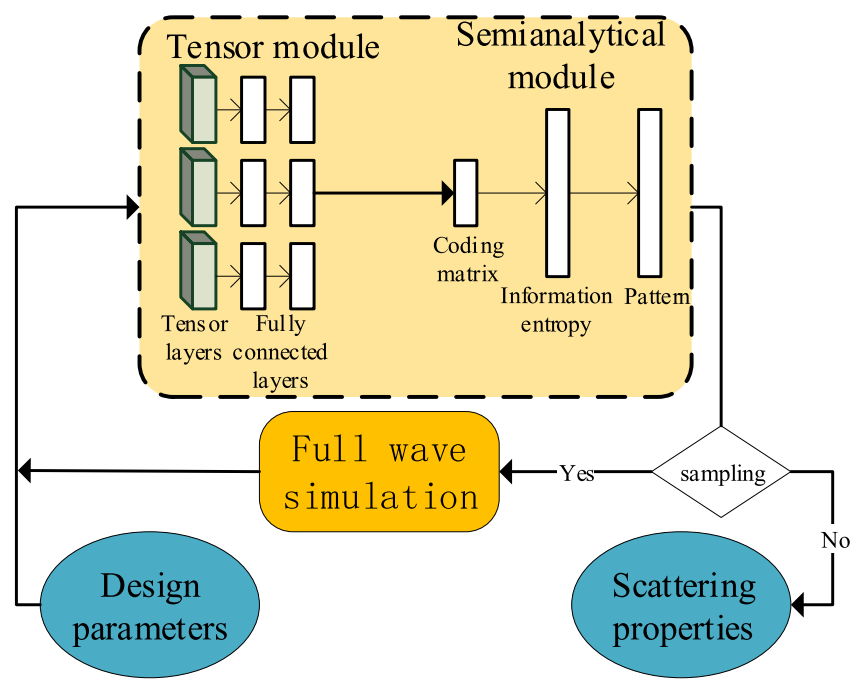

Fig. 2. Structure of the machine-learning model for designing coding metamaterials.

\section{Tailoring the scattering properties}

After analysis by semi-analytical model, we designed the unitcells to verify the model. The unitcells are composed of three-layer dielectric substrate with double metallic splitrings structure and full copper ground plane on the bottom. The dielectric substrate can be described as $\varepsilon_{r}=2.65$, $\tan \delta=0.001$, the radius of each ring is $2.5,3.8,4.1,4.6 \mathrm{~mm}$, respectively. The phase differences are approximately equal to $\pi$ in the range of $6-18 \mathrm{GHz}$ as shown in Figure 3. So, we can use the unitcells to build a metamaterial according to the coding matrix $T$.

In order to achieve the various functionalities, we used machine-learning model that shown in Figure 2 to optimize the coding matrix. We combined the scattering properties of unit cell and the semianalytical modeling and get the relationship between scattering properties and coding matrix without using full-wave simulation.

For a reflective metamaterial with $8 \times 8$ lattices, each lattice has $2 \times 2$ unitcells, the different coding matrices with different information entropy and different corresponding scattering patterns are shown in Figure 4. First we define the coding matrix $T$ as:

$$
T_{1}=\left[\begin{array}{llllllll}
0 & 0 & 0 & 0 & 0 & 0 & 1 & 1 \\
0 & 0 & 0 & 0 & 0 & 0 & 1 & 1 \\
0 & 0 & 0 & 0 & 0 & 0 & 1 & 1 \\
0 & 0 & 0 & 0 & 0 & 0 & 1 & 1 \\
0 & 0 & 0 & 0 & 0 & 0 & 1 & 1 \\
0 & 0 & 0 & 0 & 0 & 0 & 1 & 1 \\
0 & 0 & 0 & 0 & 0 & 0 & 1 & 1 \\
0 & 0 & 0 & 0 & 0 & 0 & 1 & 1
\end{array}\right]
$$

Its corresponding scattering pattern shown in Figure $4 \mathrm{a}$, we can find that the reflection of EM wave gathers in one direction. 


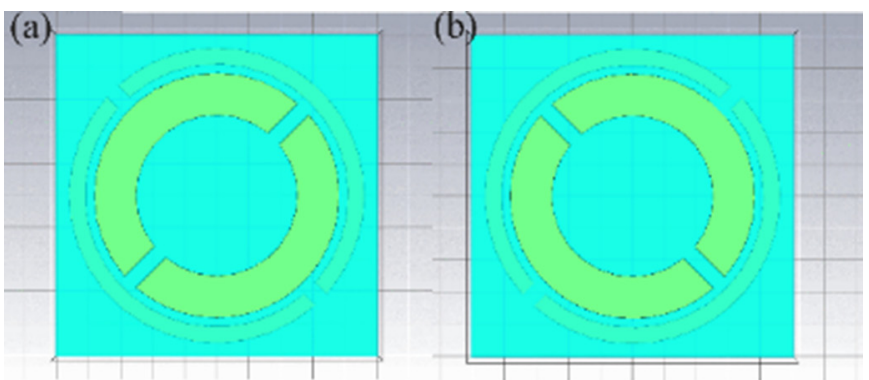

(c) S-parameters[phase]

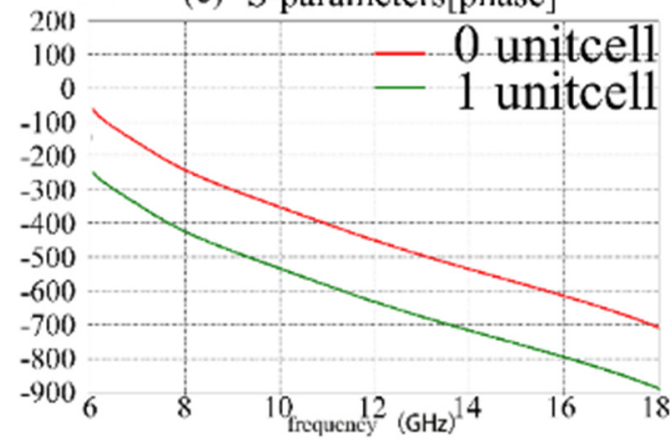

(d) phase difference

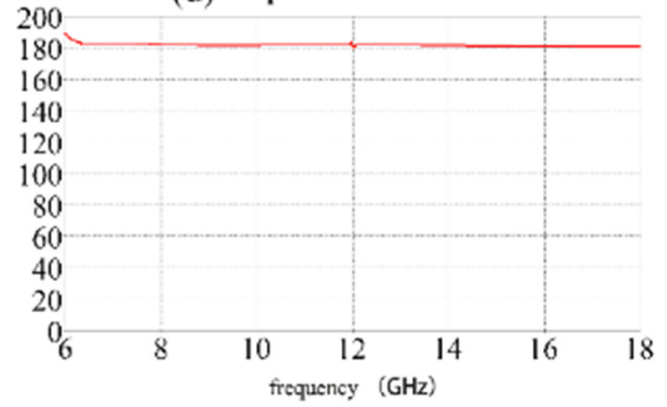

Fig. 3. Geometry of the unitcells and their reflective parameters. (a) Geometry of "0" lattice; (b) geometry of "1" lattice; (c) S-parameters of unitcells; (d) phase difference between " 0 " and "1" unitcells.

Then we try to get the maximum of information entropy. Thus, $T_{2}$ :

$$
T_{2}=\left[\begin{array}{llllllll}
0 & 0 & 1 & 1 & 0 & 0 & 1 & 1 \\
0 & 0 & 1 & 1 & 0 & 0 & 1 & 1 \\
0 & 0 & 1 & 1 & 0 & 0 & 1 & 1 \\
0 & 0 & 1 & 1 & 0 & 0 & 1 & 1 \\
0 & 0 & 1 & 1 & 0 & 0 & 1 & 1 \\
0 & 0 & 1 & 1 & 0 & 0 & 1 & 1 \\
0 & 0 & 1 & 1 & 0 & 0 & 1 & 1 \\
0 & 0 & 1 & 1 & 0 & 0 & 1 & 1
\end{array}\right]
$$

In $T_{2}$ the prior probability of " 0 " equals to the prior probability of " 1 " $(p=0.5)$, Its corresponding scattering pattern shown in Figure $4 \mathrm{~b}$, we can find that the beam of reflection divided into three direction. In other words, while the larger information entropy, the smaller radar cross section.

Furthermore, by using machine-learning to optimize the coding matrix, the coding matrix staggered alone the
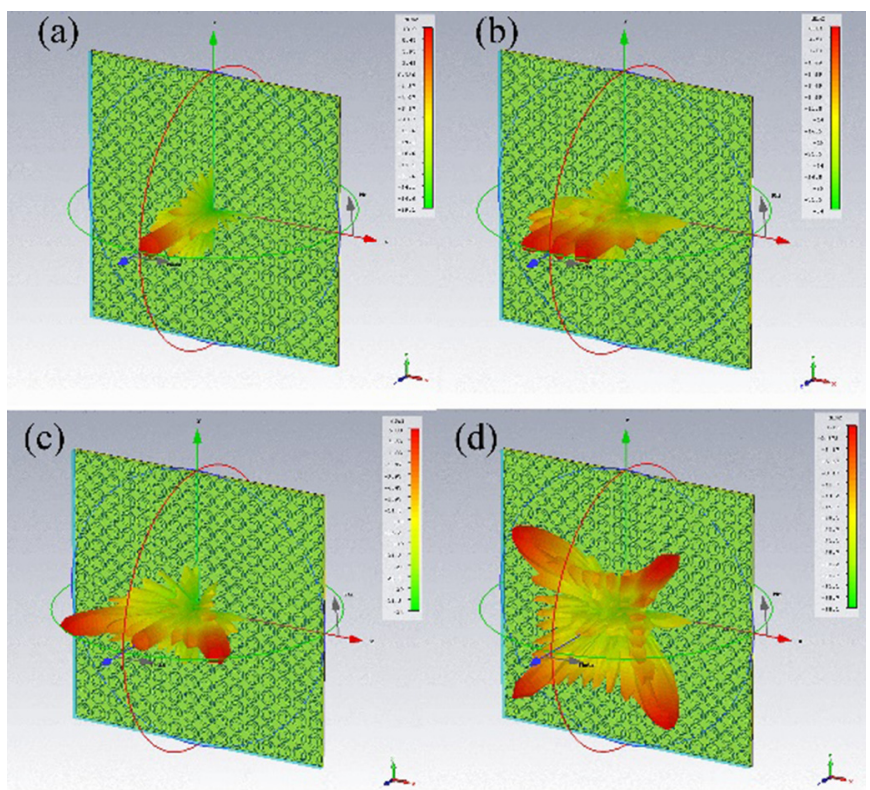

Fig. 4. Simulated scattering pattern of different coding matrices.

$\mathrm{x}$-direction, then we get the third coding matrix:

$$
T_{3}=\left[\begin{array}{llllllll}
0 & 1 & 0 & 1 & 0 & 1 & 0 & 1 \\
0 & 1 & 0 & 1 & 0 & 1 & 0 & 1 \\
0 & 1 & 0 & 1 & 0 & 1 & 0 & 1 \\
0 & 1 & 0 & 1 & 0 & 1 & 0 & 1 \\
0 & 1 & 0 & 1 & 0 & 1 & 0 & 1 \\
0 & 1 & 0 & 1 & 0 & 1 & 0 & 1 \\
0 & 1 & 0 & 1 & 0 & 1 & 0 & 1 \\
0 & 1 & 0 & 1 & 0 & 1 & 0 & 1
\end{array}\right]
$$

Its corresponding scattering pattern shown in Figure 4c, the number of "0" lattice in $T_{2}$ is same as in $T_{3}$, So the information entropy also gets the maximum value, the only difference is that the reflection is more similar to diffuse reflection in $\mathrm{x}$-direction.

Finally, we optimized the coding matrix in both $\mathrm{x}$-direction and $\mathrm{y}$-direction, and we got the fourth coding matrix:

$$
T_{4}=\left[\begin{array}{llllllll}
1 & 0 & 1 & 0 & 1 & 0 & 1 & 0 \\
0 & 1 & 0 & 1 & 0 & 1 & 0 & 1 \\
1 & 0 & 1 & 0 & 1 & 0 & 1 & 0 \\
0 & 1 & 0 & 1 & 0 & 1 & 0 & 1 \\
1 & 0 & 1 & 0 & 1 & 0 & 1 & 0 \\
0 & 1 & 0 & 1 & 0 & 1 & 0 & 1 \\
1 & 0 & 1 & 0 & 1 & 0 & 1 & 0 \\
0 & 1 & 0 & 1 & 0 & 1 & 0 & 1
\end{array}\right]
$$

Its corresponding scattering pattern shown in Figure $4 \mathrm{~d}$, compared to the results above, we can find that is a metamaterial with diffuse scattering. By using machine-learning, we encode and initialize a population, and calculated its information entropy and calculated the pattern based on the semianalytical model. Compared to the full-wave numerical simulation, the semianalytical method is easier to handle. 
We used information entropy theory to describe the metamaterial. We defined $H(X)=H\left(p_{0}, p_{1}\right)=$ $-\sum_{i=0}^{1} p_{i} \log p_{i}$ where $p_{i}$ is probability of coding matrix $X$ takes $x_{i}$. According to the entropy theory we can find that the $H\left(T_{1}\right)=0.8113, H\left(T_{2,3,4}\right)=1$. Figure 4 presents the simulation results of scattering properties. We can describe the RCS reduction qualitatively based on the number of beam dispersions.

\section{Conclusions}

In conclusion, we have investigated the design of coding metamaterials for tailor scattering properties by optimizing the coding matrix. The above results and predictions rely on semianalytical modeling and full-wave numerical simulations. They provide a way to design multifunction coding metamaterials, this may enable to approach the absolute lower RCS or shaping the EM wave. the information entropy theory and genetic optimization algorithm were used for optimized the numbers and combinational format of unitcells. From the comparison, we can tailor scattering properties by coding matrix. In addition to the reduction of RCS this method may also find more general applications to complex field.

\section{Implications and Influences}

Numerical simulation is employed to generate the scattering properties data by CST Microwave Studio. The scattering properties of interest are set in the microwave region from 6 to $18 \mathrm{GHz}$. We have collected scattering properties for training and testing. The model is constructed under the open-source machine learning framework of TensorFlow.

\section{References}

1. T.J. Cui, M.Q. Qi, X. Wan, J. Zhao, Q. Cheng, Coding metamaterials, digital metamaterials and programmable metamaterials, Light Sci. Appl. 3, e218 (2014)
2. Q. Zheng, Y. Li, J. Zhang, H. Ma, J. Wang, Y. Pang, Y. Han, S. Sui, Y. Shen, H. Chen, S. Qu, Wideband, wide-angle coding phase gradient metasurfaces based on pancharatnamberry phase, Sci. Rep. 7, 43543 (2017)

3. Q. Zhang, X. Wan, S. Liu, J.Y. Yin, L. Zhang, T.J. Cui, Shaping electromagnetic waves using software-automatically-designed metasurfaces, Sci. Rep. 7, 3588 (2017)

4. H.T. Wu, S. Liu, X. Wan, L. Zhang, D. Wang, L.L. Li, T.J. Cui, Controlling energy radiations of electromagnetic waves via frequency coding metamaterials, Adv. Sci. 4, 12 (2017)

5. J. Su, Y. Lu, H. Zhang, Z. Li, Y. Yang, Y. Che, K. Qi, Ultrawideband, wide angle and polarization-insensitive specular reflection reduction by metasurface based on parameter radjustable meta-atoms, Sci. Rep. 7, 42283 (2017)

6. Y. Shen, J. Zhang, Y. Pang, Y. Li, Q. Zheng, J. Wang, H. Ma, S. Qu, Broadband reflectionless metamaterials with customizable absorption-transmission-integrated performance, Appl. Phys. A 123, 530 (2017)

7. M. Moccia, S. Liu, R.Y. Wu, G. Castaldi, A. Andreone, T.J. Cui, V. Galdi, Coding metasurfaces for diffuse scattering: Scaling laws, bounds, and suboptimal design, Adv. Opt. Mater. 5, 1700455 (2017)

8. T.J. Cui, S. Liu, L. Zhang, Information metamaterials and metasurfaces, J. Mater. Chem. C 5, 3644 (2017)

9. K. Chen, Y. Feng, F. Monticone, J. Zhao, B. Zhu, T. Jiang, L. Zhang, Y. Kim, X. Ding, S. Zhang, A. Alu, C.-W. Qiu, A reconfigurable active Huygens' metalens, Adv. Mater. 29, 1606422 (2017)

10. K. Chen, L. Cui, Y. Feng, J. Zhao, T. Jiang, B. Zhu, Coding metasurface for broadband microwave scattering reduction with optical transparency, Opt. Express 25, 5571 (2017)

11. H. Yang, X. Cao, F. Yang, J. Gao, S. Xu, M. Li, X. Chen, Y. Zhao, Y. Zheng, S. Li, A programmable metasurface with dynamic polarization, scattering and focusing control, Sci. Rep. 6, 35692 (2016)

12. C. Huang, W. Pan, X. Ma, X. Luo, Multispectral metasurface for different functional control of reflection waves, Sci. Rep. 6, 23291 (2016)

13. K. Chen, Y. Feng, Z. Yang, L. Cui, J. Zhao, B. Zhu, T. Jiang, Geometric phase coded metasurface: from polarization dependent directive electromagnetic wave scattering to diffusion-like scattering, Sci. Rep. 6, 35968 (2016) 